Pramāña - J. Phys., Vol. 34, No. 6, June 1990, pp. 537-553. (C) Printed in India.

\title{
Generation of aperiodic tilings with fivefold symmetry by the method of intersecting decagons and diffraction from finite size tilings
}

\author{
S BARANIDHARAN, E S R GOPAL* and V SASISEKHARAN ${ }^{\dagger}$ \\ Department of Physics, ${ }^{\dagger}$ Molecular Biophysics Unit, Indian Institute of Science, Bangalore \\ 560012 , India
}

MS received 23 November 1989; revised 26 February 1990

\begin{abstract}
A method for generating aperiodic tilings with five fold symmetry is discussed here. Basic patterns formed within decagons can be used to fill two dimensional space, by matching such suitable patterns. It appears to be possible to generate perfect tilings without retracing already established coordinates imposing conditions at the initial stages of generating them. Various possible ways to generate tilings, when perfectness is not required, are discussed. The calculated diffraction patterns for some representative finite size tilings are shown. There are subtle differences in the intensities of peaks in the diffraction patterns corresponding to different finite size tilings constructed using intersecting decagons. These effects persist for a larger number of scatterers in weak peaks than in strong peaks. They are unaffected by an introduction of systematic disorder. These effects could be termed as the finite size boundary effects. There are also small shifts in the peak positions owing to the finite size effects. The possibility of formation of large approximate square cells in large tilings is shown.
\end{abstract}

Keywords. Tilings; symmetry; nonperiodic; diffraction.

PACS No $\quad 61 \cdot 50$

\section{Introduction}

The unusual interest in the quasicrystalline materials is undoubtedly due to the observation of electron diffraction spots with fivefold symmetry, normally forbidden in classical crystallography (Shechtman et al 1984). Recently, the concept of aperiodic space filling in two dimensions with fivefold symmetry has been widely discussed. The extension of the ideas to the aperiodic filling of space in 3-dimensions with icosahedral or related fivefold symmetric objects is generally considered to be the basis for understanding the experimental observations. The contact with the experimental studies of the quasicrystals is generally made through the electron or the X-ray diffraction measurements. These require a consideration of the Fourier transforms of the real space structures and have been discussed by many authors. The details of the atomic positions are as yet unknown in any of the quasicrystalline materials. Nevertheless, some aspects of the transforms are of interest, if they are explicit calculations of well defined models which could be used at least as limiting cases of experimental situations. In this article we present such aspects of the problem. A preparatory section deals with the aperiodic tilings of the 2-D plane with fivefold

*To whom correspondence should be addressed 
symmetry in a brief pedagogic way so that different results can be discussed in a compact self-contained manner.

The aperiodic tiling of a 2-D plane with 5-fold symmetry using kites and darts was first initiated by Penrose (1974) and discussed later by Mackay (1982). An algebraic analysis of Penrose tilings has been carried out by de Bruijn (1981). The possibility of tiling a plane with any given symmetry was first shown by Sasisekharan (1986) who also discussed briefly the method of intersecting decagons described in detail here. The projection from hypercubic lattices has also been a technique to produce quasiperiodic tilings (Levine and Steinhardt 1984; Kramer and Neri 1984; Kalugin et al 1985; Elser 1985; Prince 1987; Gratias and Michel 1986; Ostlund and Wright 1986). Similar tilings can also be obtained as cuts from six-dimensional lattices (Bak 1986). A general method to produce quasiperiodic patterns in arbitrary dimensions was shown by Duneau and Katz (1985). A generalised dual method for generating quasicrystal structures with arbitrary orientation was introduced by Socolar et al (1985). The diffraction properties of these tilings have been evaluated based on the reason that the tilings are of infinite size and they are obtained by a bounded projection technique. In this particle we calculate the diffraction properties of perfect Penrose tiling and other tilings, that are of finite size, generated by using the method of intersecting decagons. We show that the finite size effects are not affected by an introduction of a small amount of local disorder. When perfectness is not demanded, various tilings can be generated by different choices of the intersecting decigons.

\section{Pentagonal tiling of 2-D space}

The elementary idea of similar triangles forms a simple self similarity principle for inflation/deflation to tile the space completely. In a $\triangle \mathrm{ABC}$ with the $\mathrm{BAC}=\theta$ one can create a similar smaller $\triangle B C D$ with the $\angle B C D=\theta$ (figure 1a). This idea enables one to inflate or deflate regions of similar structures. While triangles can indeed be used as the basis for discussion, it is advantageous to use the more familiar rhombuses. In figure $1 \mathrm{~b}$, from the basic isosceles triangle $\mathrm{OAB}$ of a decagon a smaller similar $\triangle \mathrm{ABC}$ is formed. The juxtaposition of the similar triangles gives two different rhombuses. These rhombuses ACDB, OCAE may be called the acute $(\alpha, 4 \alpha)$ and the obtuse $(2 \alpha, 3 \alpha)$ respectively, where the angle $\alpha=\pi / 5$. Five of each type of the rhombuses are inscribed in the decagon. However this inscribing can be done in different ways. Four different ways A, B, C, D of arranging the rhombuses within a decagon are

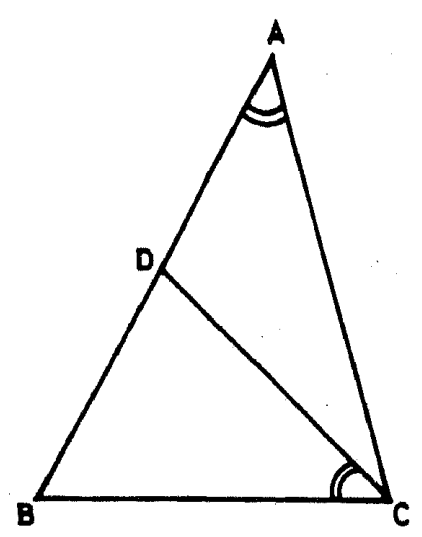

Figure 1a. The triangles $\mathrm{ABC}$ and $\mathrm{BCD}$ are similar. 


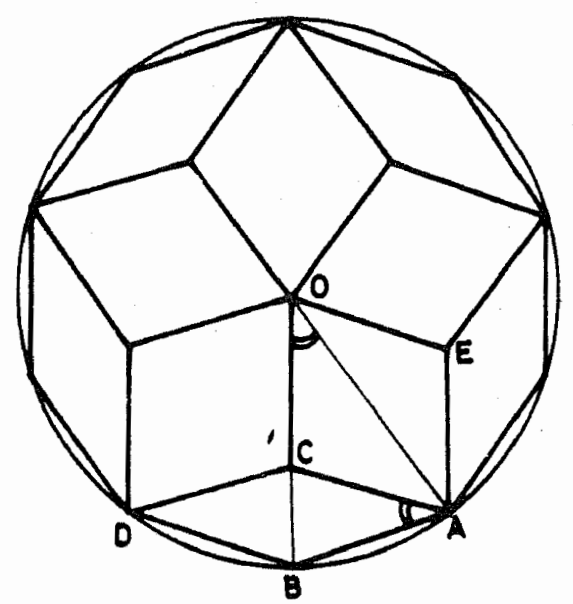

Figure $1 \mathbf{b}$. The basic isosceles triangle $A B O$ of a decagon is divided into two isosceles triangles $\mathrm{ACO}$ and $\mathrm{ABC}$ which lead to the formation of rhombuses.

A

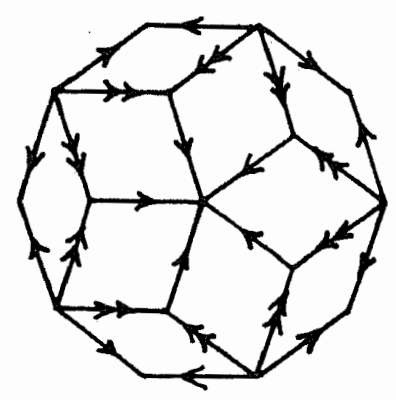

C

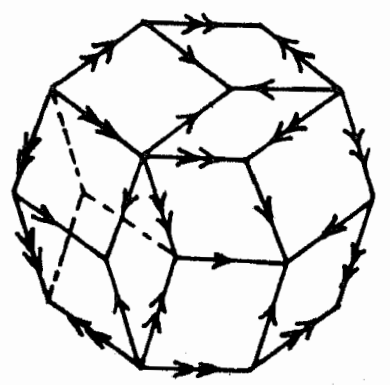

B

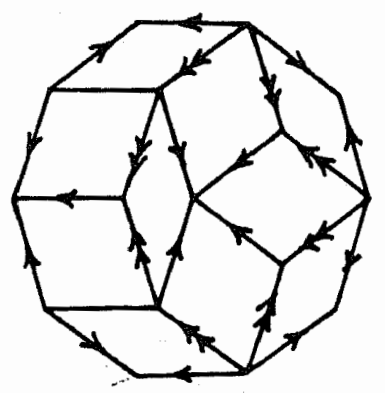

D

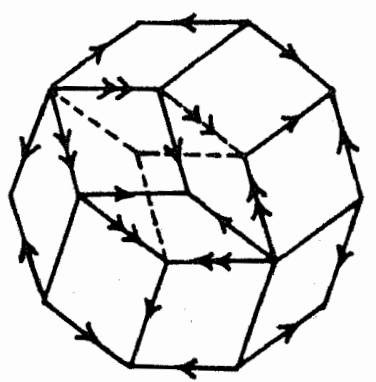

Figure 2. The four basic arrangements of the acute and obtuse rhombuses inside a decagon.

shown in figure 2. These four patterns A, B, C, D are formed so as to possess at least one mirror plane. The modifications in these patterns (shown by dotted lines) lead. to very similar results. In the case of pattern $C$ the mirror plane is destroyed during such a modification. The four different patterns A, B, C, D are shown along with the arrows indicating the Penrose matching rule. It is readily seen that the patterns $A$ and $C$ do not violate the matching rule while the patterns $B$ and $D$ do.

In order to complete the tiling of the entire plane, one proceeds to match the suitable choices of the four patterns A, B, C, D. For the clarity of illustration the decagonal patterns are inscribed in circles. It can be seen that two decagons inscribed in circles can share one, two, three or four edges such that the distance between their centres are $R 1, R 2, R 3$ and $R 4$ respectively, as shown in figure 3 . Since the radius of 

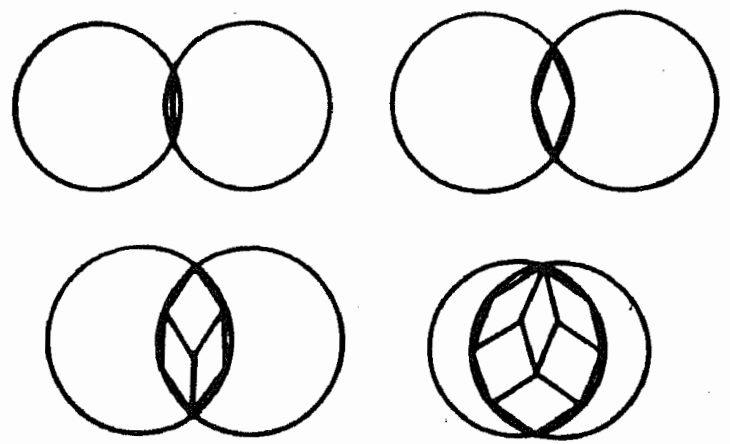

Figure 3. Two decagons inscribed in circles for clarity, can share one, two, three or four edges. Only the shared edges of the decagon are shown.
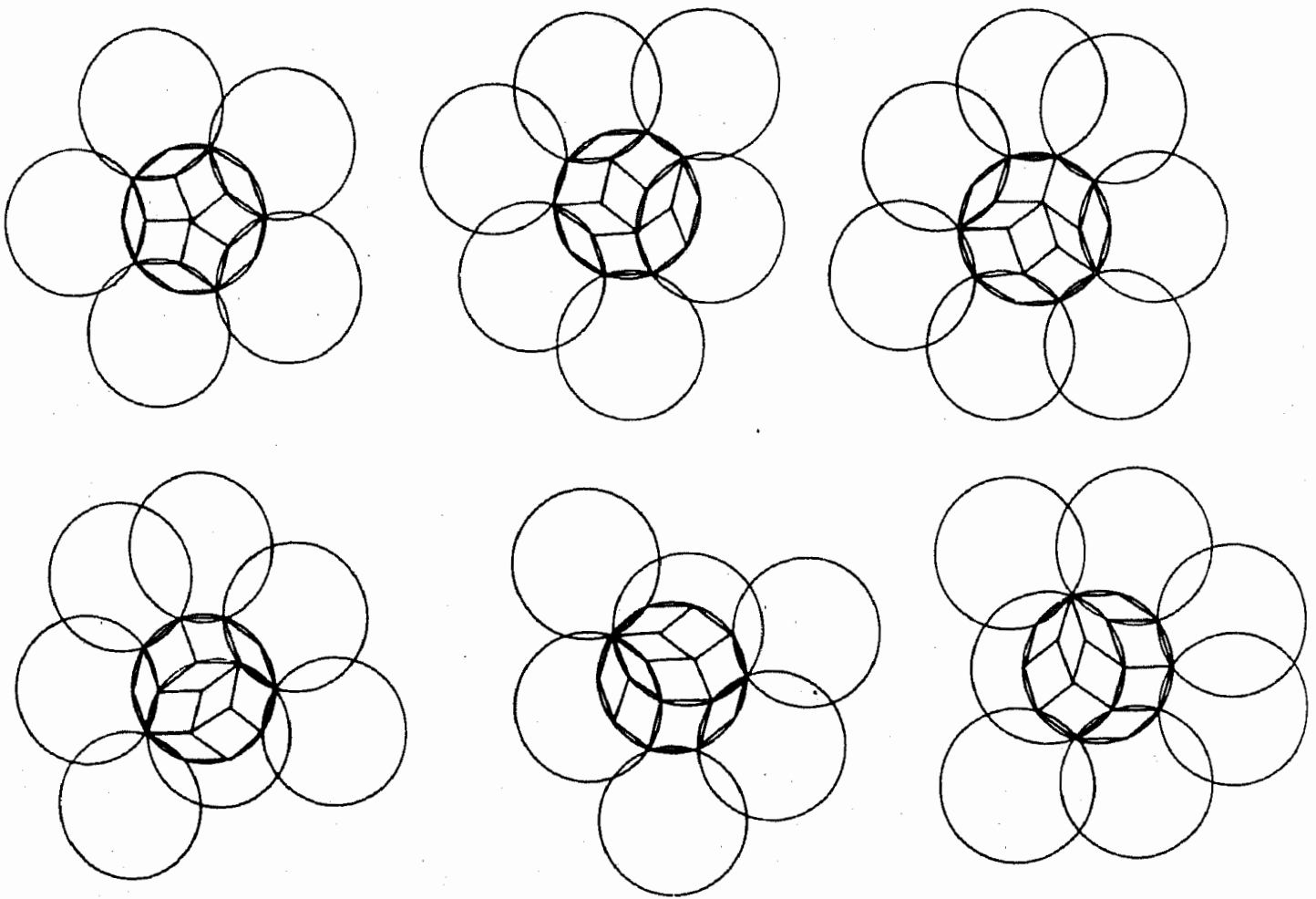

Figure 4a. The various possible sharings when more than two decagons intersect.

the circle $(R)$, the edge of the rhombus $(R / \tau)$ where $\tau$ is the golden mean) and the distance between the centres of the two circles $(R 1, R 2, R 3, R 4)$ bear fixed relationships, the sharings can easily be proved, the proof being omitted here for the sake of brevity. When two such circles intersect, the common area between them can be occupied by one edge of a rhombus, or, one acute rhombus, or, one obtuse plus two acute rhombuses or three acute plus three obtuse rhombuses depending upon the distance $(R 1, R 2, R 3, R 4)$ between the centres of the circles. Next, one sees that a circle at the centre can intersect $3,4,5,6$ or 7 circles surrounding it as shown in figure $4 a$.

By a choice of intersecting decagons it is possible to generate tilings with any combination of two or more basic patterns. As stated before basic patterns $\mathrm{A}$ and $\mathrm{C}$ do not individually violate Penrose matching rule but arbitrary combination of these two patterns cannot be expected to behave so. Nevertheless, it can be stated that an arbitrary combination of $\mathrm{A}$ and $\mathrm{C}$ patterns may contain lesser disorder than other arbitrary combinations involving $\mathrm{B}$ and $\mathrm{D}$ patterns. 


\section{Random generation of patterns}

Large tilings can be generated by computers to perform the operation recursively. In this example, the coordinates of the four basic patterns $A, B, C, D$ are initially calculated numerically, with an arbitrary choice of the coordinate axes and stored in subroutines. The pattern $A$ is chosen at the origin and the radius of the circle circumscribing it is ' $R$ '. The distance of the next intersecting circle can only be $R 1$, $R 2, R 3$ or $R 4$ (from figure 3). Starting from pattern $\mathrm{A}$ at the origin a random number is chosen from $1,2,3$ and 4 , that determines the distance $R 1, R 2, R 3$ or $R 4$ of the next pattern. In case there is no proper sharing between the two patterns, the new pattern is rotated by $18^{\circ}$ successively until all possible rotations are exhausted. The next random number is called if any rotation of the new pattern is unsuitable for matching. The coordinates of the new pattern after proper rotation is stored along with the starting pattern after eliminating repetitions. Subsequent patterns are also generated in a similar fashion such that any two patterns satisfy the possible sharings. An example of a tiling generated using basic patterns $A$ and $C$ is shown in figure $4 \mathrm{~b}$. This tiling as expected does not possess a true fivefold origin. If a true fivefold axis is to be preserved at the origin, the tiling can be generated interactively with the computer by retracing some of the already generated coordinates which in a way can be thought of as a trial and error method. At this point it is appropriate to mention that while the Penrose matching rule does not permit two adjacent rhombuses to be parallel, such a situation can arise in this method of intersecting decagons. In fact figure $4 \mathrm{~b}$ contains many local regions where two adjacent rhombuses are parallel and these configurations are termed as defects. The method of projection also does not permit this possibility (Whittaker and Whittaker 1988). The tilings with such defects cannot be classified as random (glass like) tilings because of the fact that more than

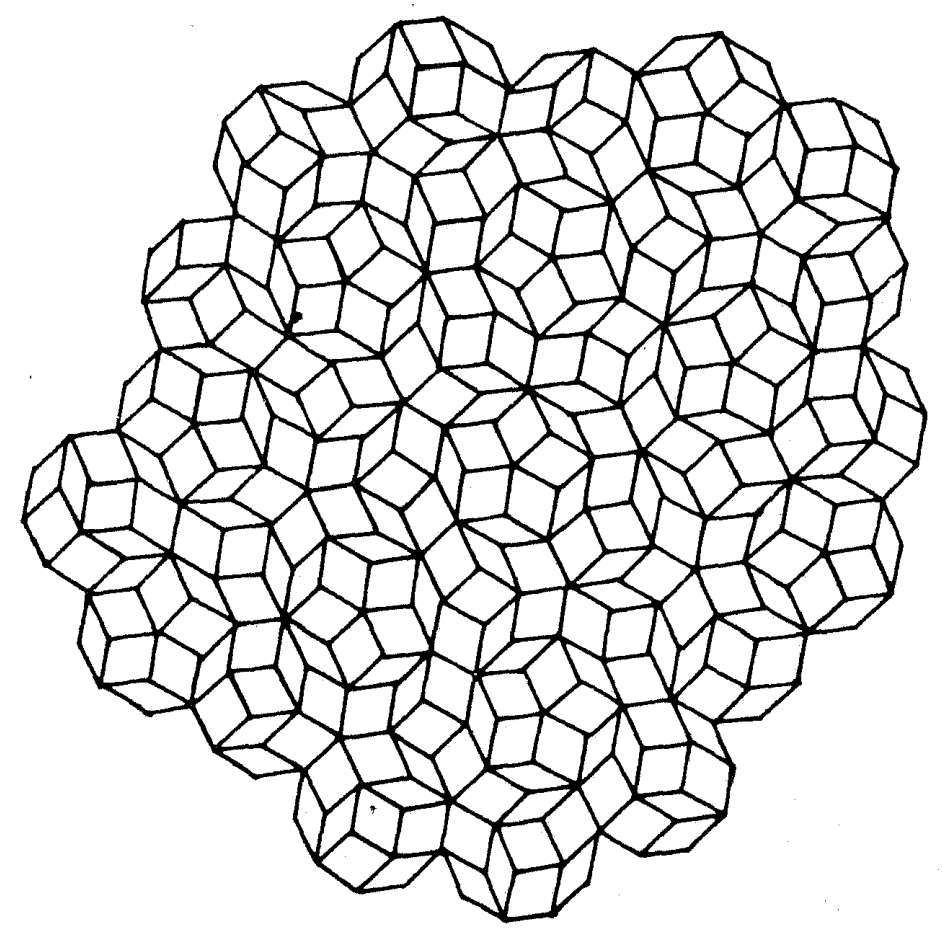

Figure $4 \mathrm{~b}$. Randomly generated tiling with $\mathrm{A}$ and $\mathrm{C}$ basic patterns. 
two adjacent rhombuses will not be parallel to each other in any situation. This is because first of all the basic decagonal patterns shown in figure 2 do not contain two adjacent, parallel rhombuses and so any possible rotation of an adjacent decagonal pattern can utmost lead to only one rhombus being parallel to another adjacent rhombus. When the tiling is constructed with $\mathrm{A}$ and $\mathrm{C}$ decagonal patterns obeying the Penrose matching rule, then the final tiling obtained belongs to the Penrose local isomorphism class. The tilings generated using the other basic patterns without having adjacent rhombuses parallel, then they can also be constructed by the generalized dual grid method. Wherever adjacent rhombuses occur in parallel, they can be considered to be localized defects, since a local modification involving any one of the rhombuses can eliminate the defect.

\section{Generation of perfect tilings}

The generation of a perfect Penrose tiling can be achieved in two ways. One will be to follow the inflation rule procedure where basic rhombuses are inflated. The other will be the tiling approach in which either rhombuses can be matched or in a similar way the basic patterns $\mathrm{A}$ and $\mathrm{C}$ can be matched. In the latter method it is necessary to retract sometimes the already generated coordinates (Steinhardt and Ostlund 1987) when the tiling is generated using a computer. One way to avoid the retracting of coordinates would be as follows: We stated earlier that not more than two rhombuses can occur adjacent parallel to each other when the tiling is made of the basic decagonal patterns. The pattern $\mathrm{C}$ has more than one possible orientation inside the decagon. Hence a proper reorientation of pattern $\mathrm{C}$ at the first stage of generation itself will result in a perfect tiling. Figure 5a illustrates the occurrence of a defect when using the decagons and also how to rectify the defect without altering the rest

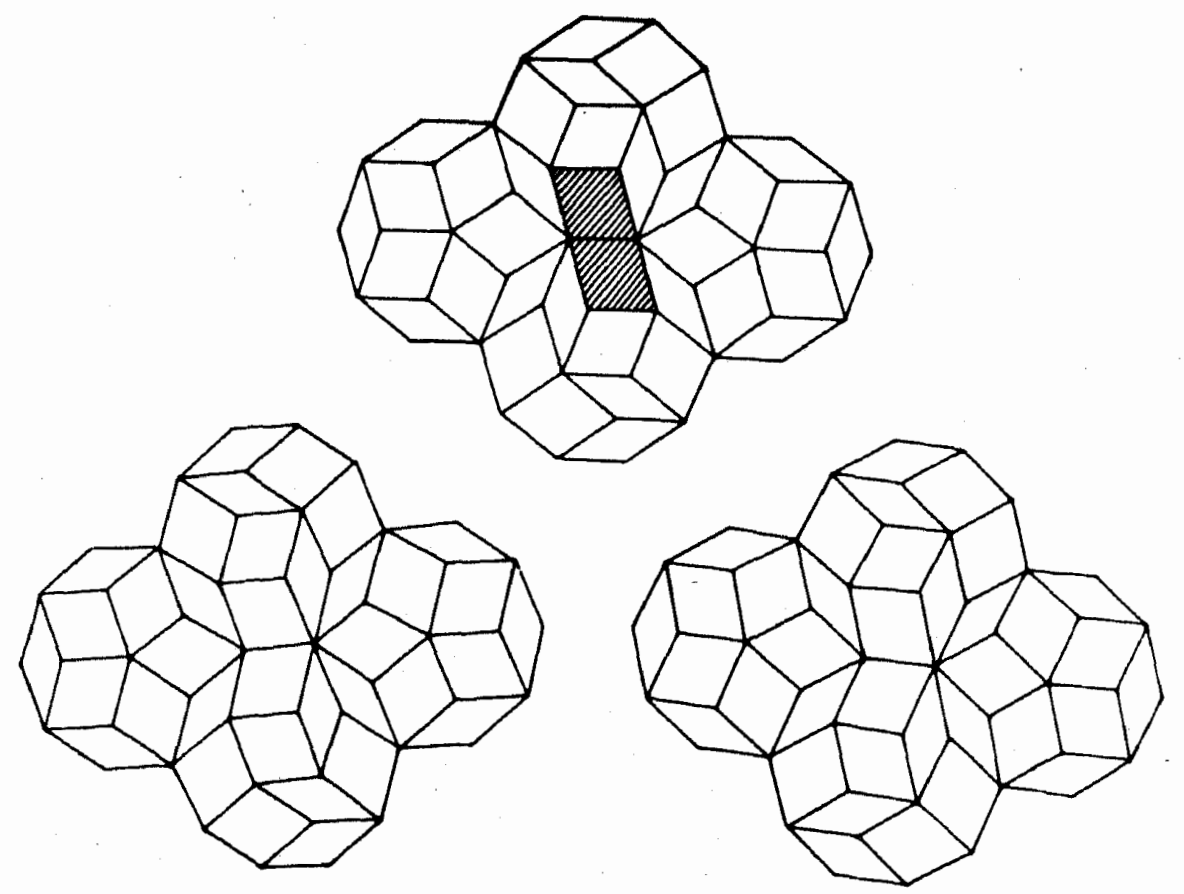

Figure 5a. Occurrence of a defect while matching patterns $\mathrm{A}$ and $\mathrm{C}$. The defect can be eliminated by rearranging a hexagon or by reorientation of a decagon as shown in the figure. 


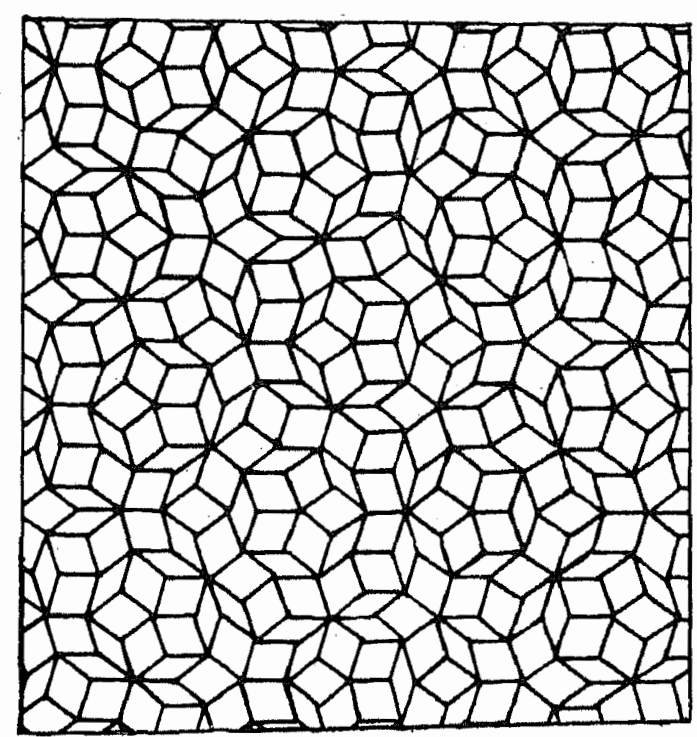

Figure 5b. A section of a perfect Penrose tiling made by matching $\mathrm{A}$ and $\mathrm{C}$ basic decagonal patterns.

of the tiling. This way to avoid defects is easily achieved with an interacting computer. Figure $5 b$ shows a section of a perfect Penrose tiling made by matching basic patterns $\mathrm{A}$ and $\mathrm{C}$ such that they obey the Penrose matching rule by reorienting the $\mathrm{C}$ pattern if and when a local defect occurred.

\section{Fourier transforms}

The Fourier transforms are needed in diffraction studies of quasicrystalline samples of the type used experimentally with electrons or X-rays. These have been calculated numerically on the basis

$$
F(h, k)=\sum_{j} f_{j} \exp \left[2 \pi i\left(h x_{j}+k y_{j}\right)\right]
$$

where $F(h, k)$ is the structure factor, $\left(x_{j}, y_{j}\right)$ are the coordinates of the scatterer with an atomic scattering factor $f_{j}$. In order to get well defined situations scatterers of unit scattering factor $\left(f_{j}=1\right)$ have been placed at the vertices of the rhombuses. The numerical method has been resorted to because of the finite nature of the tilings and due to the possible disorder in them. The analytic techniques are useful in calculating the diffraction patterns for a generic class of tilings but they cannot be used to find subtle differences arising out of disorder of the type discussed earlier as well as the finite size of the tilings.

In order to check explicitly the coherence of the scattering from various tilings, an arbitrary tiling using the basic arrangements $\mathrm{A}$ and $\mathrm{C}$ was generated (figure 6) and a circular region containing about 200 scatterers is taken. The Fourier transform of figure 6 is shown in figure 7, where a cut-off at $4 \%$ of the peak intensity has been used in the plotting to pick out the more significant peaks. In figure 7 the size of the spot corresponds to the intensity of the diffracted spot. The diffraction pattern has the fivefold symmetry as is to be expected. 


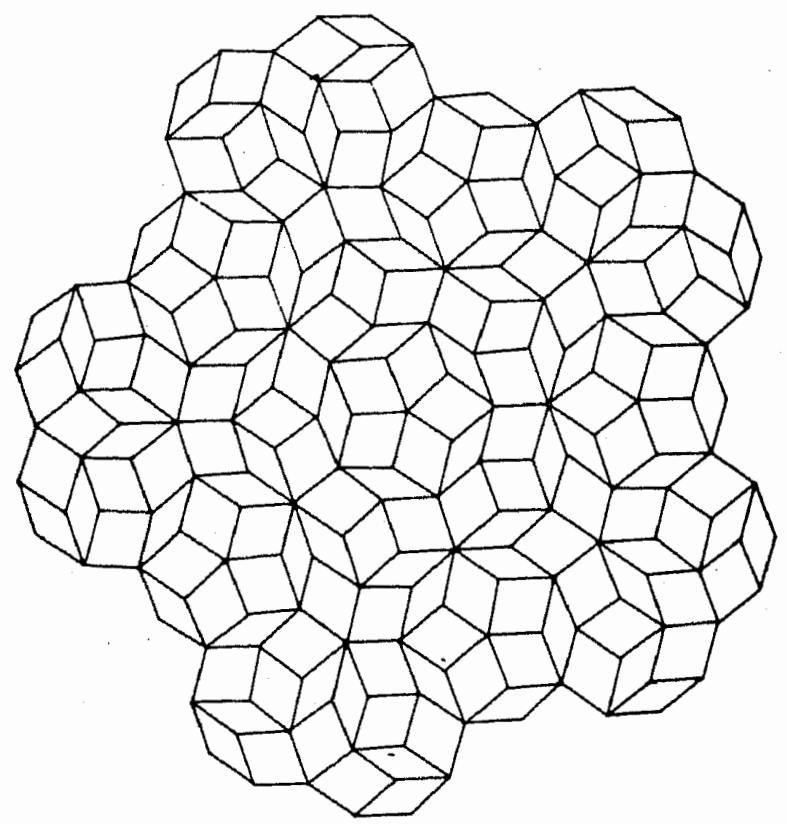

Figure 6. A small section of a tiling made of $A$ and $C$ patterns.

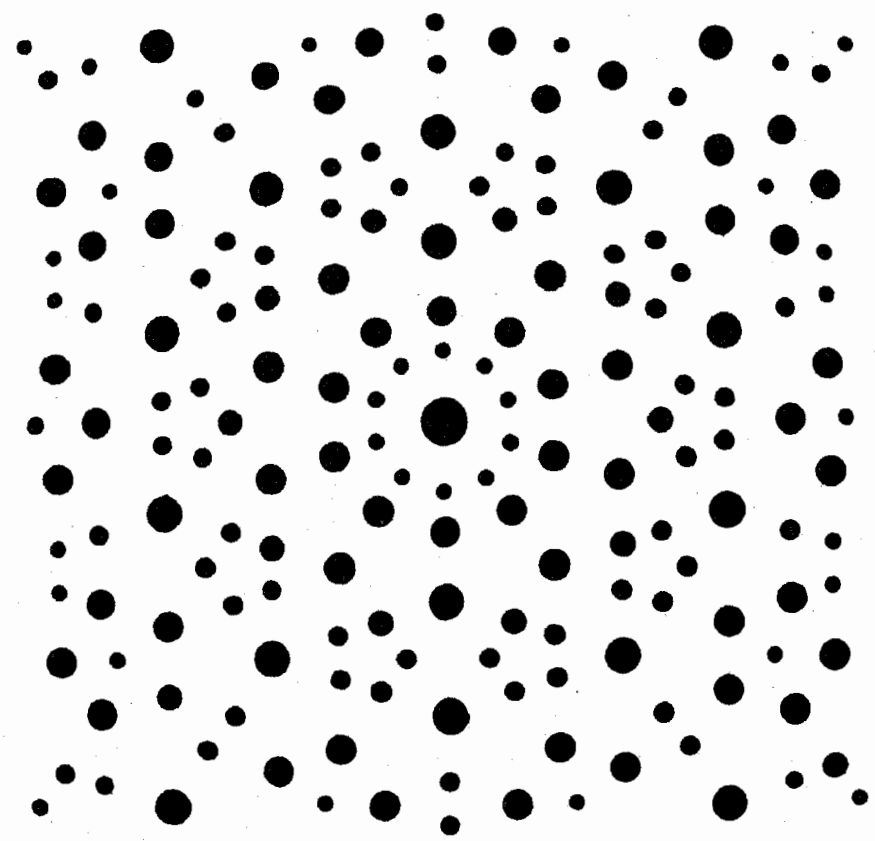

Figure 7. The diffraction pattern corresponding to the tiling shown in figure 6 .

The tiling of the 2-D plane can be done with other basic patterns as mentioned earlier. As an example, figure 8 shows the tiling where patterns $A$ and $D$ are used to generate-the tiling. A circular region containing again about 200 scatterers was chosen from the tiling and its Fourier transform was calculated. The resulting diffraction pattern is shown in figure 9 where $4 \%$ cut-off was used while plotting.

One notices specific differences though the same cut-off has been used between figures 7 and 9. In figure 7 starting from the central peak, one has two rings of 10 strong spots, followed by a third weak ring of 10 spots. However in figure 9 this third 


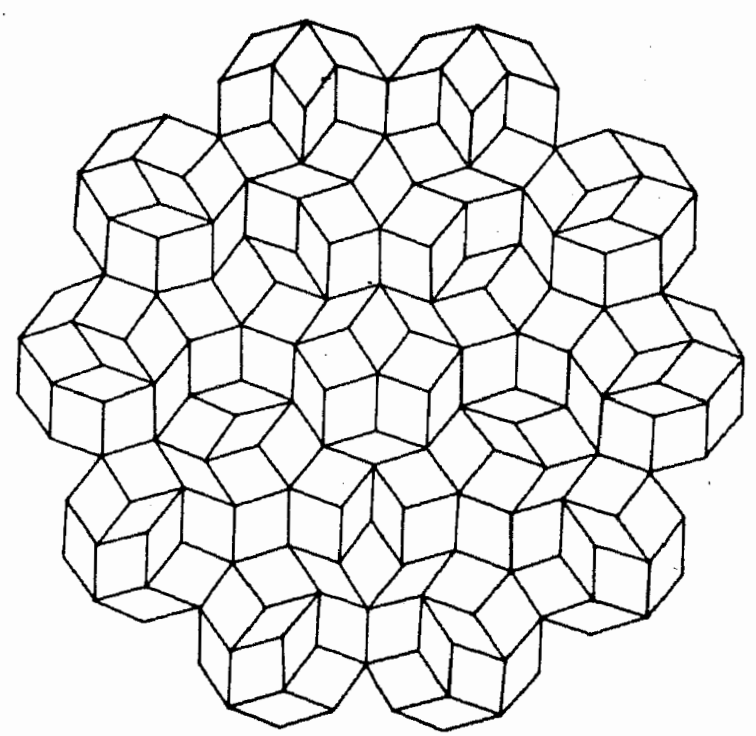

Figure 8. A small section of a tiling made of $A$ and $D$ patterns.

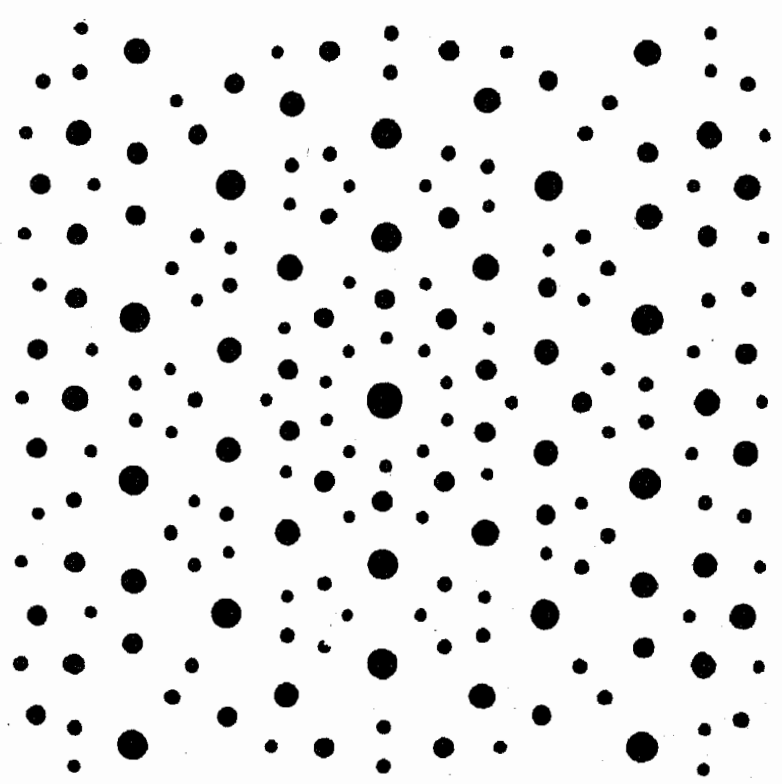

Figure 9. The diffraction pattern corresponding to the tiling shown in figure 8 .

ring of 10 spots is absent at the $4 \%$ cut-off. Their intensities are about $2 \%$ of the peak values and so are not present in figure 9, while they are about $10 \%$ in figure 7 . Again the pentagonal symmetrically located spots have different intensities. These differences could be interpreted as arising out of the finite nature of the tilings. A comparison of figure 7 and figure 9 with figure 10 which is an electron diffraction pattern taken from a fivefold axis of a grain of Al-Mn icosahedral phase shows that the agreement is better between figure 7 and figure 10 .

It is also of great interest to see the nature of scattering from different sections of the same tiling. Figure 11 shows the circular region of a tiling with $\mathrm{A}$ and $\mathrm{C}$ patterns. This figure possesses a true fivefold axis at the origin and more than 1000 scatterers. Figure 12 shows the diffraction pattern of figure 11 with $1 \%$ cut-off. Figure 13 shows the circular region of a tiling again with $\mathrm{A}$ and $\mathrm{C}$ patterns, but the true fivefold 


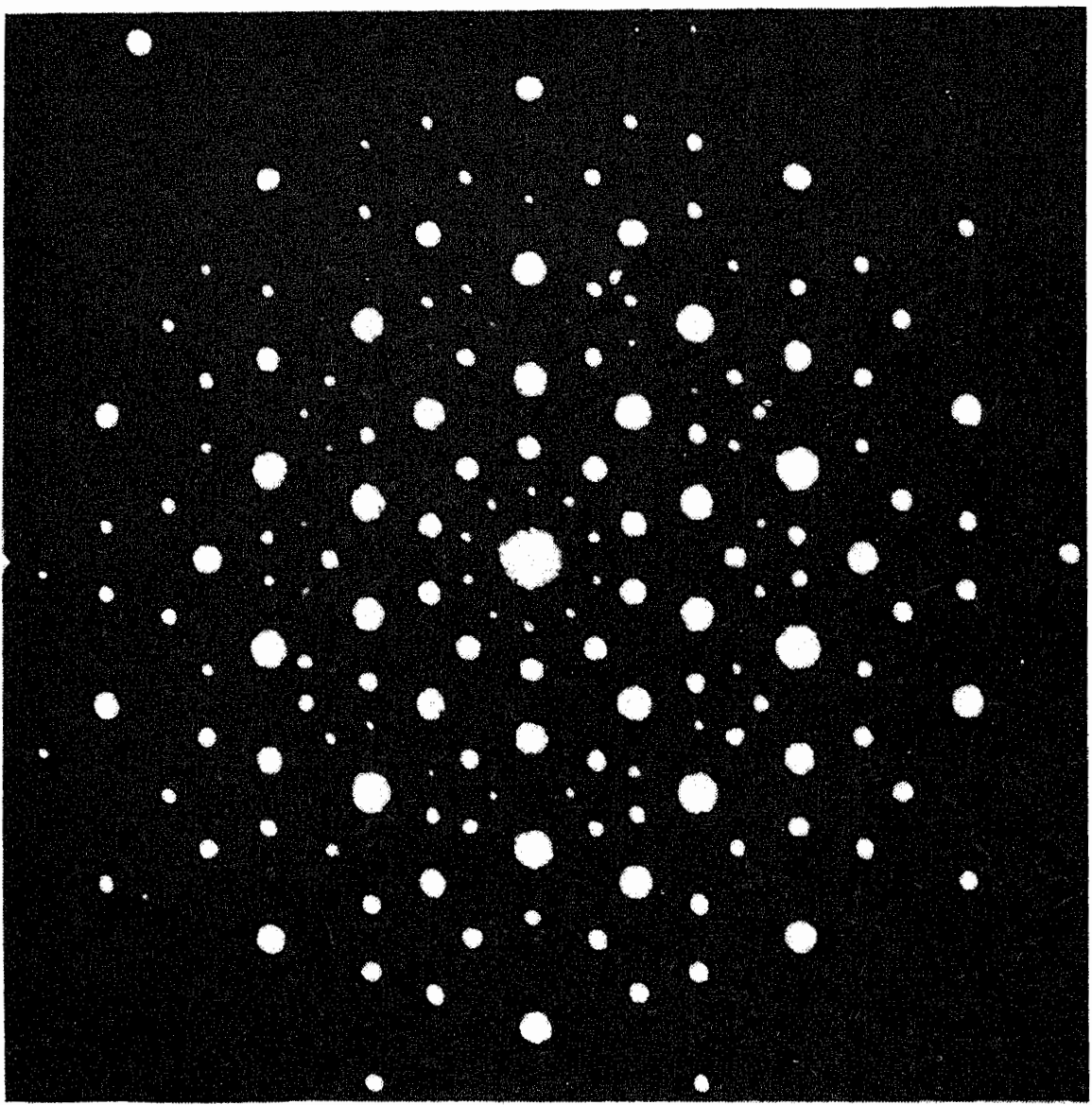

Figure 10. The electron diffraction pattern taken from a grain of $\mathrm{Al}-\mathrm{Mn}$ icosahedral phase. Compare the position and intensity of the peaks with those in figure 7 and in figure 9 .

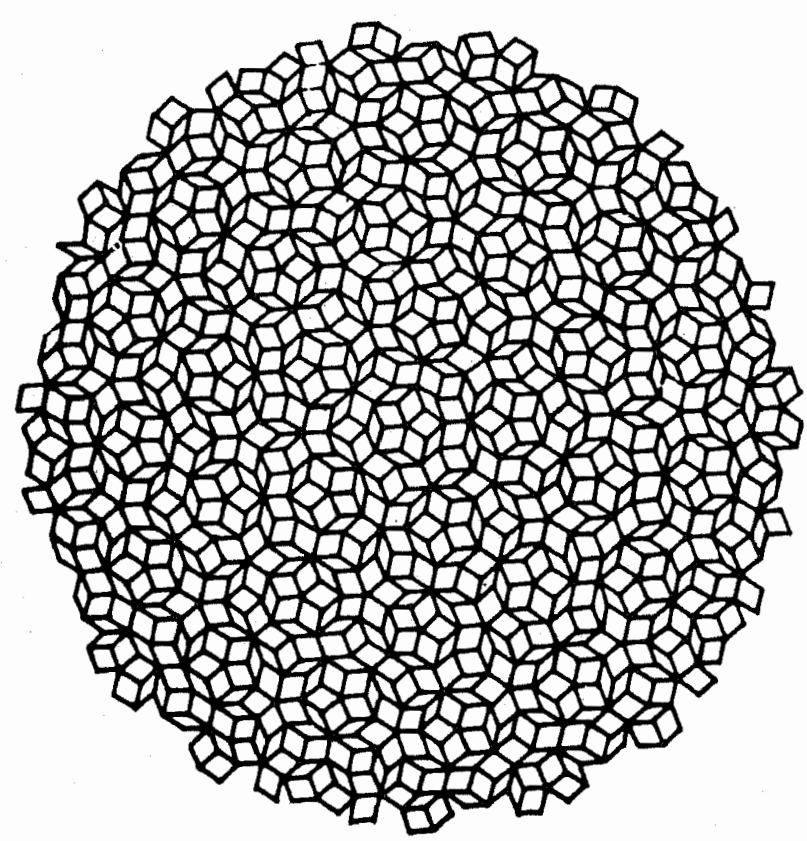

Figure 11. An aperiodic tiling with true fivefold symmetry at the origin with nearly 1000 verticies. 


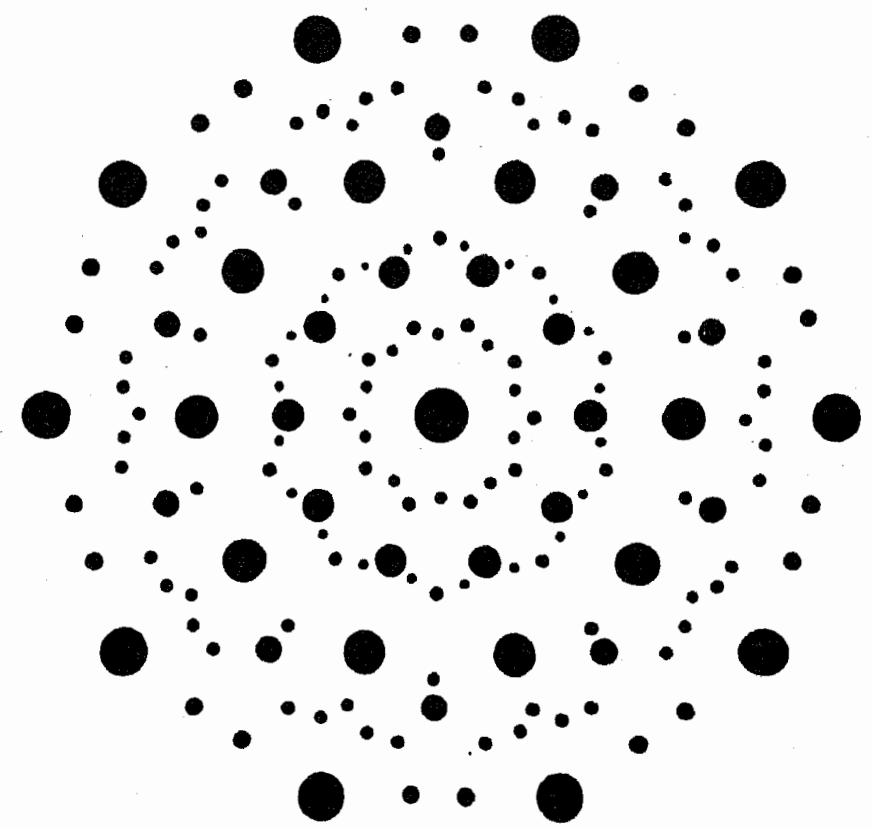

Figure 12. Diffraction pattern corresponding to the tiling shown in figure 11.

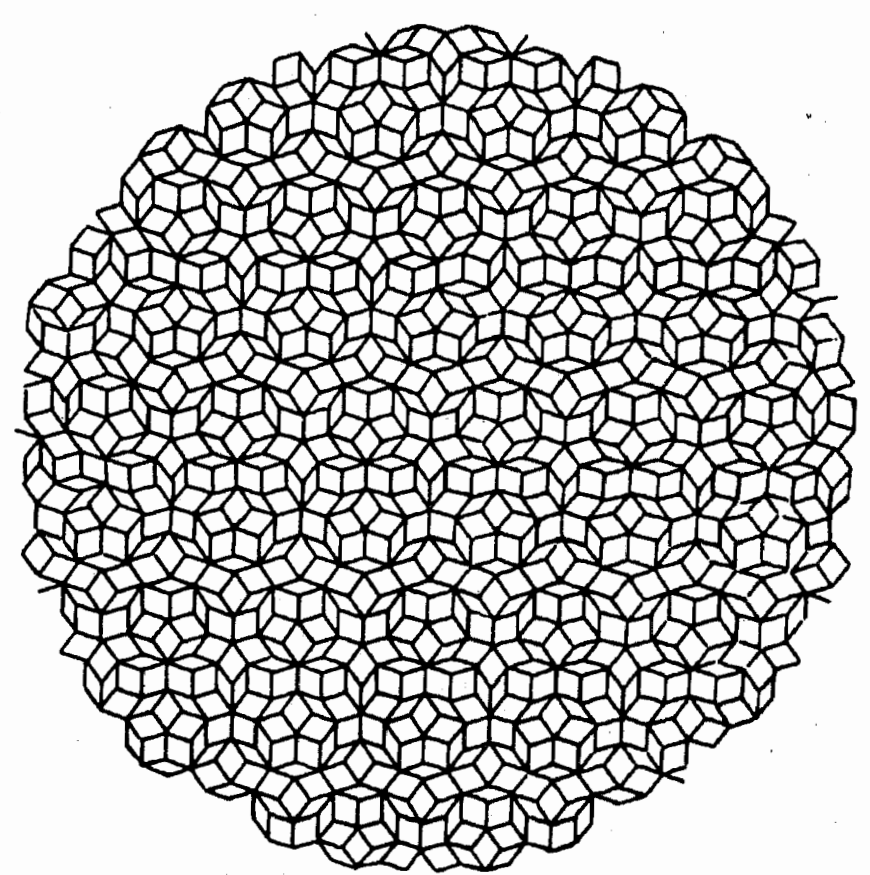

Figure 13. An aperiodic tiling without the true fivefold symmetry origin and with nearly 1000 vertices.

symmetry axis is destroyed. The true fivefold axis can be considered to be at some other arbitrary point outside the tiling. The calculated diffraction pattern of this tiling is not significantly different from the diffraction pattern (figure 12) although there are some small differences in the intensities of very weak spots. This implies that the location of the true fivefold axis is not significant as regard to the coherency of scattering. 


\section{Finite size effects}

A study of finite size effects becomes important because one of the relevant models for quasicrystals is the icosahedral glass model (Stephens and Goldman 1986). If the icosahedral clusters possess a orientational order then the diffraction from the quasicrystalline phase can be considered to be as a result of diffraction from individual clusters. While the particle size effects are known for crystalline phases, such effects have not been evaluated so far for quasicrystal models.

The finite size effects on some selected peaks have been calculated by varying the number of scatterers. These effects have been calculated for the tiling with true fivefold symmetry axis (figure 11), for the tiling without the true fivefold symmetry axis (figure 13) and for the perfect Penrose tiling shown in figure 5b. These effects for the strong and weak peaks have been calculated by following the intensity of the peak, $I(N)$ as a function of the number of scatterers, $N$. Figure 14 shows the behaviour of the intensity of various peaks as a function of $N$. In all cases there are fluctuations when the number of scatterers is less and the oscillations disappear for large values

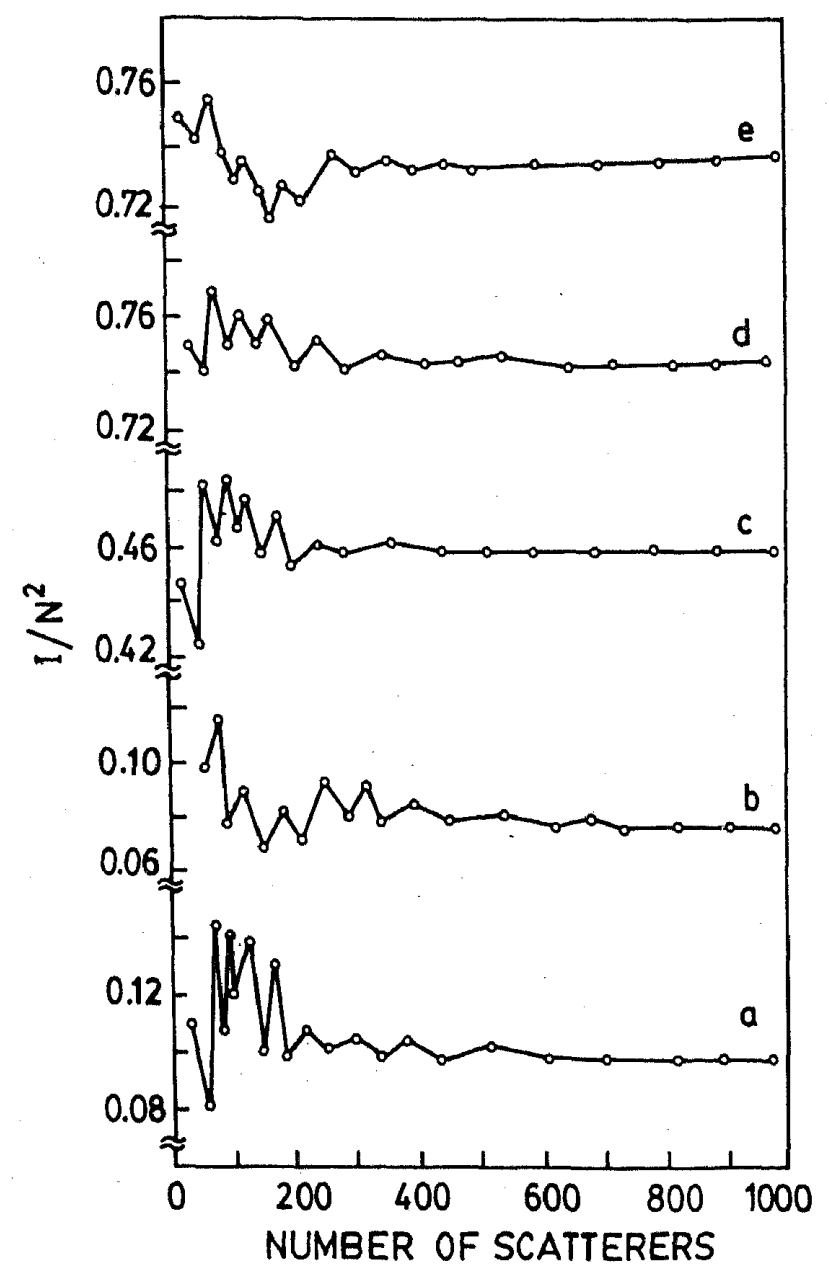

Figure 14. (a) The finite size effects on the intensity of a weak peak from the diffraction from figure 11 ; (b) the finite size effects on the intensity of a weak peak for figure 13 ; (c) the finite size effects on the intensity of a strong peak from the diffraction from the perfect Penrose tiling shown in figure $5 \mathrm{~b}$; (d) the finite size effects on the intensity of a strong peak for figure 11 ; (e) the finite size effects on the intensity of a strong peak for figure 13 . 


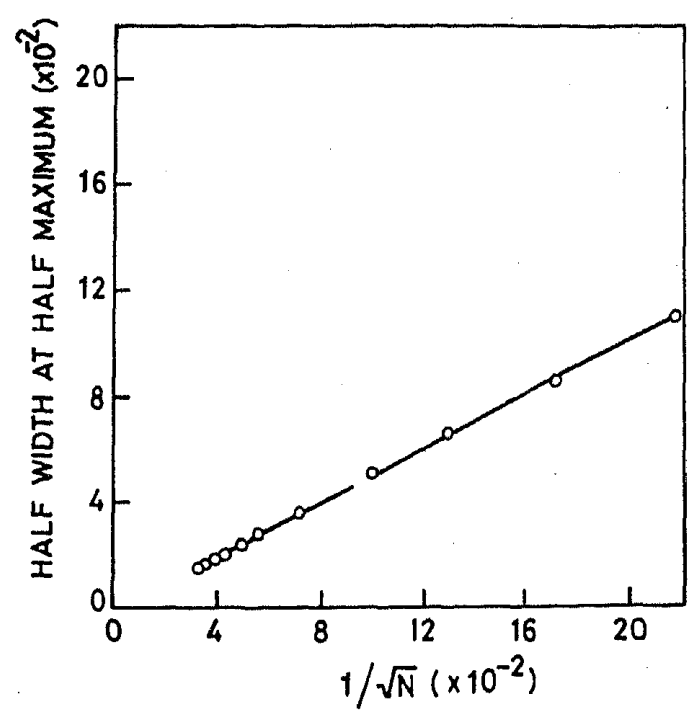

Figure 15. The half width at half maximum as a function of the number of scatterers.

of $N$. In the case of weak peaks the size effects last for a slightly larger number of scatterers $(N \sim 400)$ as compared to the strong peaks where the effects are present up to $N \sim 300$. Also seen from figure 14 is that the size effects are present for the perfect Penrose tiling. It is clear from this figure that defects in a small proportion do not affect the intensity of the peaks as much as the size effects do.

The peak broadening of the same peak considered in the case of the perfect tiling has been monitored as a function of number of scatterers. Figure 15 shows the variations of the half width at half maximum of the peak as a function of $1 / \sqrt{N}$. The slope of the straight line is an analogue of the familiar Scherrer constant. The HWHM is found to be just inversely proportional to the number of scatterers along the reciprocal vector considered very similar to the cases of crystalline materials. The experimental observation of large peak widths in quasicrystal diffraction may possibly support the icosahedral glass model whe $e_{\perp}+$ the individual icosahedral clusters could actually resemble a small tiling with a small number of scatterers. The shifts in a peak position (Socolar et al 1986) have also been evaluated as a function of the number of scatterers for a strong peak in the diffraction from the perfect tiling. It is seen from figure 16 that the shifts in a mean peak position become negligible when $N$ goes above 300 .

Another way of studying the finite size effects is to study the diffraction side-lobes in the fine structure of the shape of the spots. Figures 17 and 18 show the scan of the $h$-axis for $N=100$ and $N=500$ referring to the diffraction in figure 12 . The sharpening of the peaks and the movements of the side-lobes towards the peak when the number of scatterers is increased, is a direct reflection of the effect of the finite size of the tiling and the coherence among the contributions from the different scatterers. The sharpening of the peaks and the movements of the side-lobes also indicate a necessity to locate the exact peak positions for every $N$. The exact peak positions (within an absolute error of $10^{-5}$ have been located for every $N$ in all the above analyses by maximising the intensity until any further refinement in the peak positions does not affect the intensity values significantly. We have shown qualitatively that there are shifts in peak positions and fluctuations in the intensity of peaks due to finite size of the tilings irrespective of the perfect or imperfect nature of the tiling. 


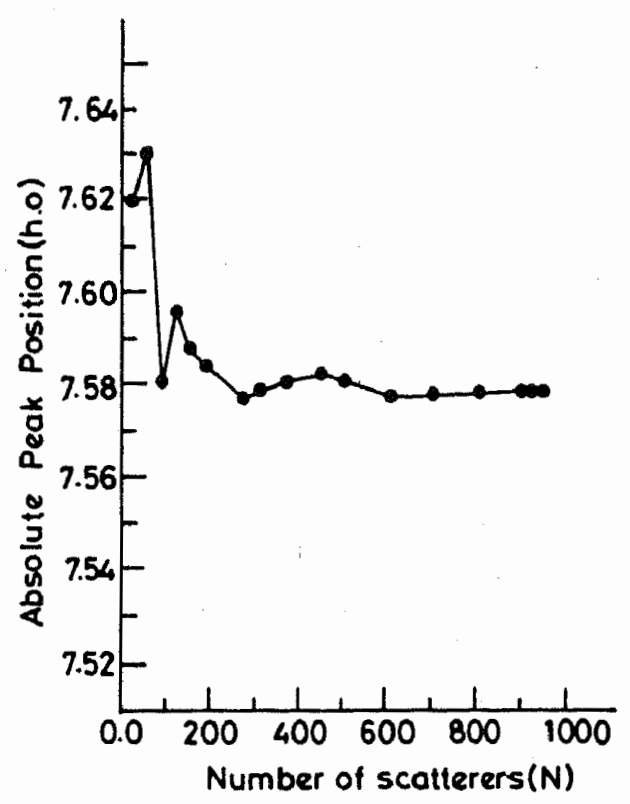

Figure 16. Shifts in a peak position as a function of number of scatterers.

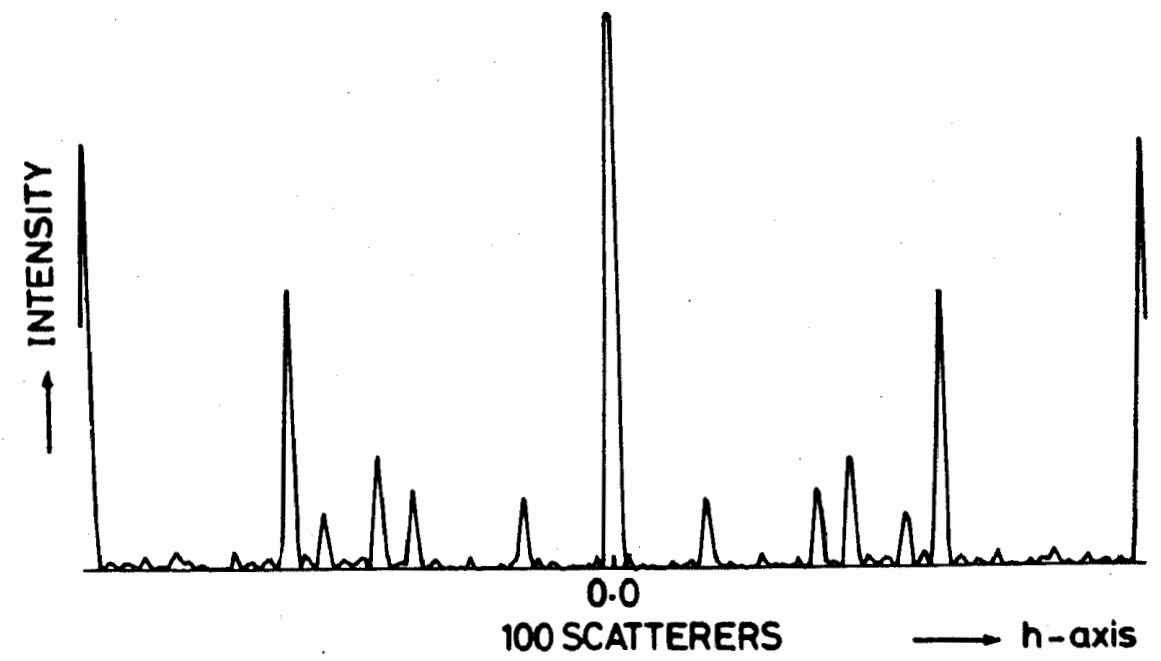

Figure 17. Scan of $h$-axis of figure 12 for $N=100$.

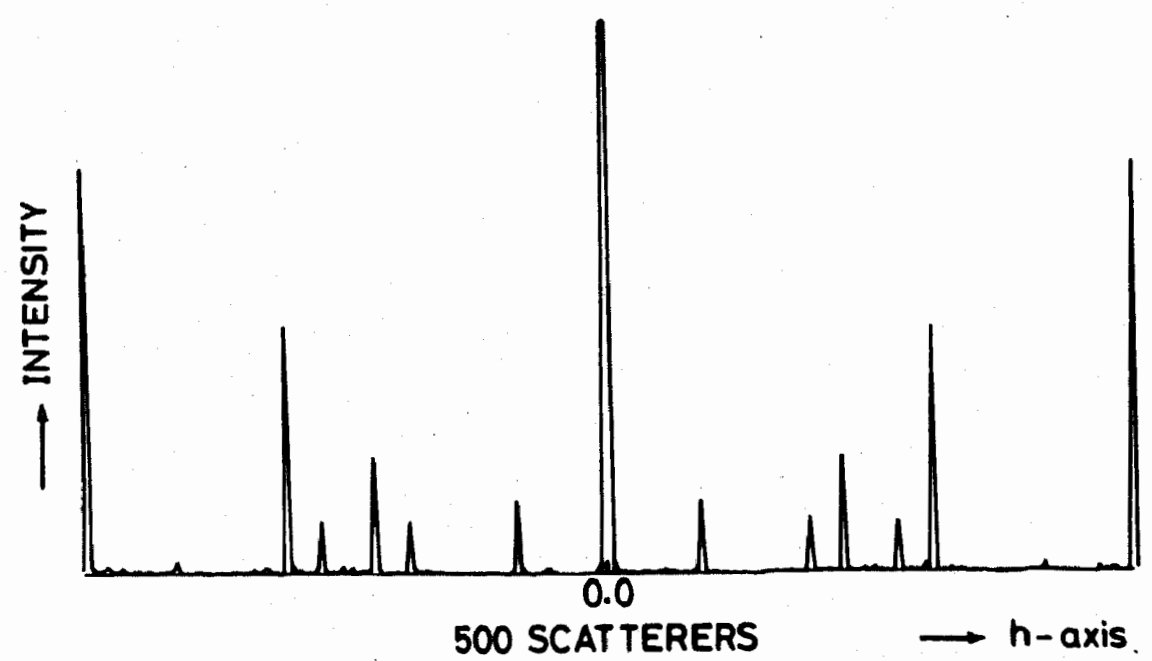

Figure 18. Scan of $h$-axis of figure 12 for $N=500$. 
Clearly the peak shifts, defects and dislocations, half widths of the peaks, peak profiles and other intensity statistics are also of interest. These will be reported in due course.

\section{Formation of nearly square lattices}

It was pointed out by Sasisekharan (1986) that pentagonal tilings can have regions of apparent, approximate square symmetry. This can be seen from figure 19 where a tiling with $\mathrm{A}$ and $\mathrm{C}$ basic patterns is drawn, showing the true fivefold symmetric origin and the extended region. The thick lines connecting the centres of the local fivefold symmetries form a hexagonal pattern. On the extended region of figure 19 one has marked with broken lines, the approximate square cells. One side of the cell is larger than the other side by $2 \cdot 5 \%$. The angles formed by the broken lines are exactly $90^{\circ}$. On extending the drawing further it is possible to have larger cells of approximate square shape with lesser error. Given the ion sizes of $\mathrm{Al}$ and $\mathrm{Mn}$, the cell shown in figure 19 has an edge of about $30 \AA$, very comparable in size to the cells proposed by Pauling (1987) in the twinning model of quasicrystals. The Fourier transforms of the vertices from the square regions of figure 19 is shown in figure 20. The number of vertices within the square regions is $\sim 300$.

The basic patterns used in figure 19 are $\mathrm{A}$ and $\mathrm{C}$. Therefore the diffraction pattern (figure 20) should be compared with figure 12 which is the Fourier transform of the tiling in figure 11 containing basic patterns $\mathrm{A}$ and $\mathrm{C}$. In figure 20 the arrowed spots arranged in a twofold configuration are slightly more intense $(1 \%)$, although not visually observable, than the other spots in the same layer. The vertices inside the approximate square region in figure 19 are themselves arranged with a mirror symmetry and hence the diffraction pattern shows a deviation from figure 12 . Also we earlier stated that the finite size effects tend to settle down for $N>300$. In order to determine if the boundary effects are persistent significantly for $N>300$, a set of 4 such approximate square cells $(N \sim 1150)$ adjacent to each other has been considered

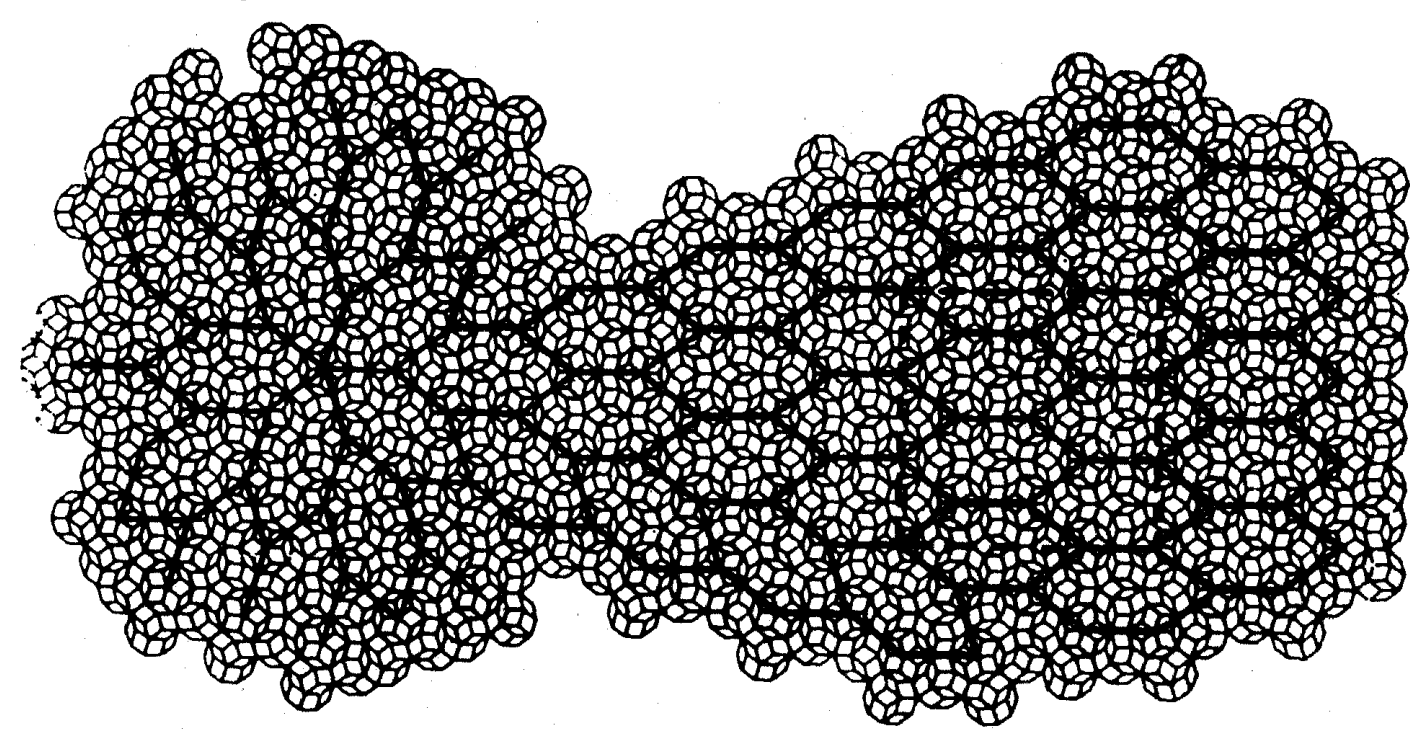

Figure 19. A large section of an aperiodic tiling with fivefold symmetry and the extended region. Also observe the hexagonal superstructure and the approximate square cell marked by the dotted lines. 


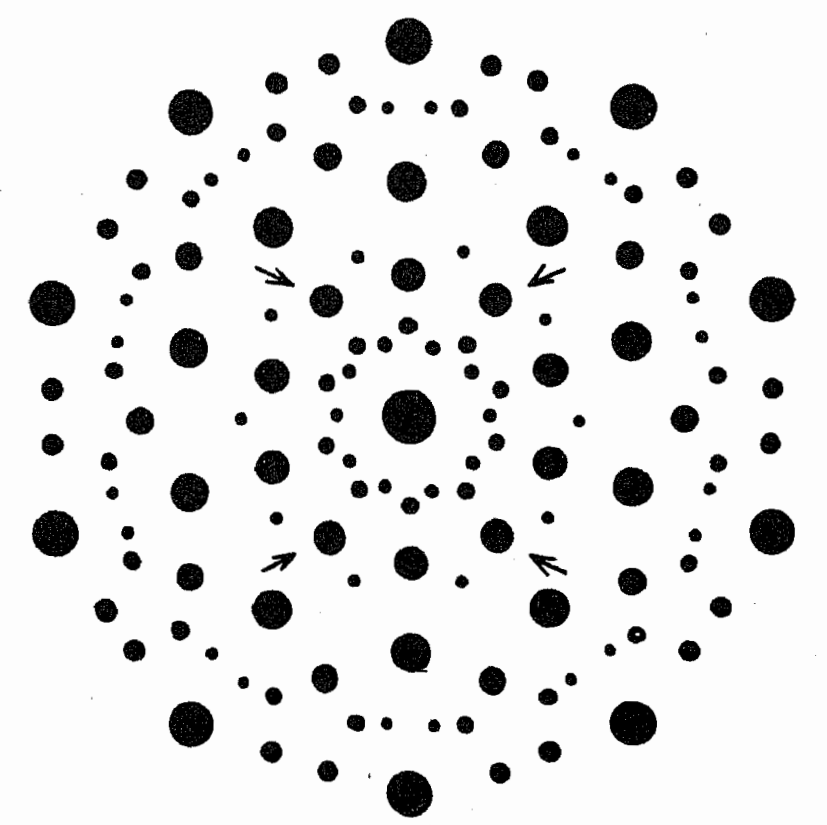

Figure 20. The computed diffraction pattern of the contents of the approximate square cell marked in figure 19.

and its Fourier transform was calculated. The resulting diffraction pattern was very similar to figure 12 within computational error. Hence we conclude that the diffraction from approximate square cells could bear resemblance to the diffraction from truly aperiodic structures. In the absence of standard methods to determine the structure of the quasicrystals either in the form of rhombohedrons or in the form of icosahedral clusters, it may be possible to approximate the structure to a near cubic symmetry and followed up experimentally.

\section{Acknowledgements}

The authors are grateful to Prof. S Ramaseshan and Prof. R Nityananda of Raman Research Institute, Bangalore for their comments and discussions on Penrose tilings. The suggestions and assistance of Messrs. V S K Balagurusamy, T Narasimhamurthy, $M$ Sundaramoorthy and other colleagues have been invaluable and we thank them for the same. We also thank Prof. M A Viswamitra for his interest in our work on tilings and X-ray diffraction from quasicrystals. We thank the referee for explaining the peak broadening nature and for suggesting other improvements. This work is part of the research carried out on quasicrystals funded by the Department of Science and Technology, Government of India.

\section{References}

Bak P 1986 Scr. Metall. 201199

de Bruijn N G 1981 Proc. Ned. Acad. Wet. Ser. A84 39

Duneau M and Katz A 1985 Phys. Rev. Lett. 542688

Elser V 1985 Phys. Rev. B32 4892

Gratias D and Michel L 1986 J. Phys. (Paris) 47 C3 
Kalugin P A, Kitayev A.Yu and Levitov L S 1985 J. Phys. Lett. 46 L601

Kramer P and Neri R 1984 Acta Crystallogr. A40 580

Levine D and Steinhardt P J 1984 Phys. Rev. Lett. 532477

Mackay A L 1982 Physica A114 609

Ostlund S, Wright D C 1986 Phys. Rev. Lett. 562068

Pauling L 1987 Phys. Rev. Lett. 58365

Penrose R 1974 Bull. Inst. Math. \& its Appln. 10266

Prince E 1987 Acta Crystallogr. A43 393

Sasisekharan V 1986 Pramana - J. Phys. 26 L283

Shechtman D, Blech I, Gratias D, Cahn J W 1984 Phys. Rev. Lett. 531951

Socolar J E S, Steinhardt P J and Levine D 1985 Phys. Rev. B32 5547

Socolar J E S, Lubensky T C, Steinhardt P J 1986 Phys. Rev. B34 3345

Steinhardt P J, Ostlund S 1987 The physics of quasicrystals (Singapore: World Scientific)

Stephens P W and Goldman A I 1986 Phys. Rev. Lett. 561168

Whittaker E J W and Whittaker R M 1988 Acta Crystallogr. A44 105 